\title{
Ratzke, Christian (202I). Hochschuldidaktisches Interreligiöses Begegnungslernen. Eine empirisch-explorative Studie zum Potenzial interreligiöser Kompetenzentwicklung in der Ausbildung von Ethik- und Religionslehrer_innen (Internationale Hochschulschriften 686). Münster: Waxmann. ISBN 978-3-8309-44I 5-7. 260 Seiten.
}

\section{Norbert Brieden}

Bergische Universität Wuppertal (brieden@uni-wuppertal.de)

Die religiöse und weltanschauliche Pluralität nimmt weiter zu. Damit wächst auch die Bedeutung interreligiösen Lernens. Wie wird hochschuldidaktisch in der Ausbildung von Ethik- und Religionslehrer*innen auf diese Anforderung reagiert? Ratzke untersucht Chancen und Grenzen des Interreligiösen Begegnungslernens, wie es an den Standorten der KPH Wien/Krems und der PH Heidelberg praktiziert wird. Dazu hat er in seiner von Christoph Knoblauch (PH Ludwigsburg) betreuten Dissertationsschrift zehn leitfadengestützte Telefoninterviews transkribiert und ausgewertet: vier mit Studierenden, die am interreligiösen Begegnungstag der KPH Krems am 17.12.2016 teilnahmen (die katholische Probandin und der evangelische Proband waren über 40 Jahre alt, die beiden muslimischen Proband*innen Anfang bis Mitte 20); sechs mit Studierenden, die den interreligiösen Studientag der PH Heidelberg am 4.7.2017 besuchten (die jüdische Probandin war 53 Jahre alt und nahm als einzige bereits zum zweiten Mal am Studientag teil, die beiden christlichen Probandinnen (evangelisch und katholisch), die beiden muslimischen Proband*innen und die Probandin, die das Fach Philosophie studiert, waren Mitte bis Ende 20).

Flankiert wurde die qualitative Stichprobe erstens durch eine quantitative Fragebogen-Analyse, in der vor und nach den interreligiösen Begegnungen Kompetenzen der „religiösen Identitätssicherheit“ (sechs Items: 201) und der „vorurteilsbewussten Haltung“ (zehn Items: 210) eruiert werden sollten (96 Studierende haben an beiden Befragungen teilgenommen). Zweitens sollten die Hypothesen der Studie durch Interviews mit fünf am Projekt des Begegnungslernens im WiSe 2018/19 beteiligten Dozierenden der PHs Karlsruhe und Heidelberg (evangelische Theologie: Uta Schmidt und Eva Korneck; katholische Theologie: Herbert Stettberger; muslimische Theologie: Imran Schröter; Ethik/Philosophie: Hans-Bernhard Petermann) verifiziert oder falsifiziert werden (131).

Bevor Ratzke das Forschungsdesign (112-152) und die Ergebnisse der empirischen Untersuchung (153239) darstellt, führt er in die Rahmenbedingungen und (religions-)pädagogischen Begriffsbestimmungen des interreligiösen (Begegnungs-)Lernens und verschiedener Kompetenzmodelle sowie den Studienaufbau an den beiden untersuchten Lernorten ein (15-111). Am Ende dieser theoretischen Grundlegung steht ein Kompetenzstruktur-Modell mit sieben Kompetenzen (teilweise differenziert nach Niveaustufen), das im Abgleich mit der Auswertung der empirischen Daten entstand (wobei das Verhältnis von induktivem und deduktivem Vorgehen eigens zu reflektieren wäre): 1. Religiöse Wahrnehmungskompetenz; 2. Kompetenz der Anerkennung; 3. Von der Differenzkompetenz zur Ambiguitätstoleranz; 4. Reflektierter Perspektivenwechsel; 5. Empathiekompetenz; 6. Religiöse Identitätssicherheit; 7. Vorurteilsbewusste Haltung (110f.).

Diese sieben Kompetenzen strukturieren dann die Darstellung der Forschungsergebnisse: die ersten fünf im Blick auf die zehn Interviews (156-198), die letzten beiden in der Analyse der quantitativen Befragung (199-221) und alle sieben in der "Schlussbetrachtung“, die zur Bestätigung Aussagen aus den 
Interviews mit den Dozierenden und aus der im ersten Teil dargestellten religionspädagogischen Literatur einbezieht (222-238). Die aus dem empirischen Material gewonnenen zwölf Forschungshypothesen bieten wenig Überraschendes, können aber doch die zuvor bereits vermutete positive Wirkung des interreligiösen Begegnungslernens für die Anbahnung der sieben Kompetenzen bestätigen (238f.), auch für die Gruppe der Ethik- und Philosophiestudierenden, die allerdings nicht mit „Konfessionslosen“ gleichgesetzt werden sollten (220), da Konfessionalität und Philosophiestudium einander nicht ausschließen.

Insgesamt ist Ratzkes „Forscherreflexion“ zuzustimmen, dass seine Studie „lediglich explorative Aussagen zulässt“" (239), die zu weiterer Forschung , an der Performanz interreligiöser Kompetenzen“ motiviert (245). Denn überraschend war für ihn „die Exponiertheit prozessbezogener Kompetenzen“, was ihn erkennen ließ, „, dass Wissen allein nicht zu Performanz interreligiöser Kompetenzentwicklung führen kann" (244) - eine nicht unbekannte Einsicht zur Diskrepanz von Wissen und Handeln. Auch scheint mir der Begriff der „Kompetenzanbahnung“ durch interreligiöses Begegnungslernen bescheidener und zutreffender als der Begriff der „Kompetenzentwicklung“. Denn sollte die Studie eine solche Entwicklung nachweisen wollen, wäre sie nicht valide. Dieser Eindruck wird zuweilen erweckt, etwa wenn Ratzke aufgrund der „Auswertung der quantitativen Daten“ feststellt, „dass die Lernenden [...] interreligiöse Kompetenzentwicklungen im Bereich der religiösen Identitätssicherheit und der vorurteilsbewussten Haltung“" zeigten (219).

Aber kann etwa das im Posttest festgestellte Gefühl, das Bekenntnis der eigenen Religionsgemeinschaft sei eher bestätigt worden im Vergleich zu der weniger hohen Erwartung zu einer solchen Bestätigung im Pretest (F4: 201) bei allen untersuchten Lerngruppen (202-209) wirklich eine solche Kompetenzentwicklung bezüglich der Identitätssicherheit belegen? Verweist nicht die geringere Erwartung einer solchen Bestätigung eher auf die Offenheit, mit der die Studierenden freiwillig in das interreligiöse Begegnungslernen gehen? Umgekehrt ergäbe sich die paradoxe Vermutung: Wer erwartet, sich in keiner Weise verunsichern zu lassen (und damit zeigt, dass sein Glaube starr ist), könnte durch die Begegnung mit einem fremden Glauben eher verunsichert werden. Vielleicht ist eine solche Befürchtung auch eine unbewusste Ursache für das von einem Studierenden aus Wien angemerkte und kommentierte Fehlen einer Studierendengruppe: „Die orthodoxen Studienkollegen haben das abgelehnt an dieser Begegnung teilzunehmen. Das ist ja auch vom Bischof verboten worden. Ja. Die dürfen nichts Interreligiöses da machen" (275).

Und wenn die Zustimmung zu vermuteten Reibungspunkten im Pretest höher sind als zu den erlebten Reibungspunkten (210): Wieso ist das dann ein Indiz für die Kompetenzentwicklung in der vorurteilsbewussten Haltung? Spricht das nicht einfach dafür, dass die Studierenden, die sich am interreligiösen Begegnungslernen beteiligten, eben nicht die vermuteten Vorurteile hegten? Inwiefern lässt sich aus der Diskrepanz von Erwartung und Erfahrung eine Kompetenzentwicklung ableiten? Ein Indiz für solche Fehlschlüsse könnte sein, wenn Ratzke die Aussagen von „Paul“ (evangelisch, Wiener Gruppe) als „Vorurteile“ interpretiert (165). Aber sind die negativen Erfahrungen Pauls mit einem muslimischen Religionslehrer an seiner Wiener Vorort-Schule bereits Vorurteile? Wenn Paul erlebte, dass dieser türkische Lehrer mit niemandem aus dem Kollegium sprach, den Schüler*innen trotz ständiger Abwesenheit „einen Dreier" gab (276), ist es dann korrekt, ihm „eine Angst vor dem Fremden, vor dem bislang nicht Greifbaren“ (165), zu unterstellen? Wenn Paul sich nun von der Offenheit der muslimischen TheologieStudierenden so begeistern lässt, dass er einen von ihnen später um Rat zu einem „Unterrichtsentwurf“ (196) bzw. zum Entwurf einer interreligiösen Feier fragt (276), sind dann bezüglich seiner Empathie wirklich Abstriche aufgrund mitschwingender „Vorurteile“ zu machen (195)?

Interessant wäre, die in den Interviews ausgedrückten inhaltsbezogenen Kompetenzen, die „als notwendige Grundlage interreligiösen Lernens verstanden werden“ (244), in ihrem Verhältnis zu dem vo- 
rausgesetzten Kompetenzstrukturmodell zu erörtern, ohne sich dabei auf die durch religionskundliches Wissen unterstützte „Entwicklung religiöser Wahrnehmungsfähigkeit“ zu beschränken (224). Zu wünschen wäre, dass der online zur Verfügung gestellte Anhang neben den angekündigten aber dort fehlenden Interviewleitfäden (136f., Anm. 95f.) auch noch die Transkripte der Interviews mit den Dozierenden zur Verfügung stellte - vielleicht könnte das unkompliziert noch ergänzt werden.

Insgesamt leistet Ratzke mit seiner Studie wichtige Pionierarbeit, die zu weiterer Forschung motiviert, etwa zum Verhältnis von Kompetenzniveau- und Kompetenzentwicklungsmodellen, die Kompetenzstrukturen nach unterschiedlichen Kriterien differenzieren. 